\title{
NEW INVENTIONS
}

\section{MAGNETIC SEPARATION DEVICE}

Inventors: J.E. Foy and E.B. Sweeney

Applicant: Dexter magnetic Technologies

Patent no.: US 6,413,420

Publication date: 2 July 2002

A magnetic biological particle separation device comprising a permanent magnet structure substantially surrounding a vessel containing a liquid and a biological sample in colloidal suspension in the liquid described. The magnetic separation device comprises a window through which to view the liquid. The permanent magnet structure comprises a plurality of permanent magnets arranged in such a manner to provide for superpositioning of the external magnetic field generated by each magnet. The external magnetic field acts on magnetic beads attached to the biological sample, causing the sample to move toward the inner wall of the vessel. The permanent magnets do not completely surround the outside of the vessel to allow the liquid to be poured out of the vessel.

\section{PROCESS FOR PRODUCTION OF MAGNETI LIQUID}

Inventors: V.M. Makarov et al.

Assignee: ITET, Yaroslavskiy G. Tekh. Univ.

Publication date: 10 May 2003

Patent no.: RU 2182382

Invention is related in particular to synthesis of key component of magnetic liquid highly dispersed magnetite. Process of production of magnetic liquid includes formation of magnetite suspension by way of mixing of hydroxide $\mathrm{Fe} 2+$ won from pickling solution by precipitation with ammonium hydroxide and of hydroxide $\mathrm{Fe} 3+$, coating of surface of magnetic particles by adsorbing layer of stabilizing substance, heating up of suspension of magnetic particles with layer of stabilizing substance adsorbed on them, separation of fraction carrying stabilized magnetic particles in liquid carrier from suspension. Hydroxide of trivalent iron formed in the capacity of waste of clearing of sewage of electroplating shops is utilized as hydroxide Fe3+ to produce magnetic ferrophase. 


\section{FILTER APPARATUS WITH MAGNETIC SEPARATION}

Inventor: J.R. Bratten

Applicant: Filtra Systems Company

Patent no.: EP1123741, A3

Publication date: 16 August 2001

A filter incorporates a magnetic separator comprised of a series of side by side but spaced apart permanently magnetized bars interconnected to form a train, movably mounted through a tank into which is introduced liquid contaminated with ferromagnetic particles to be removed. The bar train includes a section overlaying a vacuum box in the tank which also is overlain with a filter media to remove particles not removed by the separator. The bar train may be mounted to a flight conveyor included in the filter, or alternatively have its own support and drive. A scraper station is located at a discharge end of the tank for removal of accumulated particles from the bars.

\section{APPARATUS AND METHOD FOR USE IN FERROHYDROSTATIC SEPARATION METHOD AND APPARATUS}

Inventor: J. Svoboda

Applicant: De Beers Consolidated Mines, South Africa

Patent no.: WO0048740

Publication date: 24 August 2000

The invention concerns, in one aspect, a ferrohydrostatic separation method in which a ferrofluid is used to separate materials of different density. In the method, the apparent density of the ferrofluid is controlled by means of a vertically orientated magnetic field generated by a C-dipole, open dipole (O-dipole) or a split pair electromagnet or permanent magnet. Other aspects of the invention include an apparatus for use in this method and a process for separating materials of different density using the method.

\section{METHOD OF CONCENTRATION OF RARE EARTH ORES}

Inventor: I.M. Petrov

Patent no.: RU2182521

Publication date: 20 May 2002

A rare-earth ore is ground and then subjected to first stage of magnetic separation, thus obtaining heavy magnetic product, medium magnetic middlings and non-magnetic product. Heavy and medium magnetic fractions are classified, then subjected to additional magnetic separation. Weakly magnetic product from the first stage is combined with weakly magnetic fraction from the second stage of magnetic separation to form rare-earth concentrate. The first stage of magnetic separation is performed at the magnetic field of $1.5 \mathrm{~T}$ while the second stage at 1.5 to $2 \mathrm{~T}$. 


\section{METHOD AND APPARATUS FOR MAGNETIC} SEPARATION OF PARTICLES

Inventors: G. Hatch and M. Schilling

Applicant: Dexter Magnetic Technologies

Patent no.: WO02066165

Publication date: 29 August 2002

A method and apparatus for magnetic separation of particles within a container. In one embodiment, a container contains a number of particles and a number of magnetic susceptible particles. A number of magnets are arranged in a plane and is placed close to the container. The magnetic poles of the magnet are arranged in a pattern to apply magnetic fields oriented perpendicular to the plane on the container. The pole pattern provides consistent separation, across the container, of the number of magnetically susceptible particles from the rest of the particles. 